\title{
Simple Evaluation of the Influence of an Inert Additive on the Swelling Characteristics of Clay Soil
}

\author{
Bahia LOUAFI ${ }^{1}$, Ramdane BAHAR ${ }^{2}$ \\ Laboratory of Environment, Water Geomechanics and Structures (LEEGO), \\ Faculty of Civil Engineering USTHB, Algiers, Algeria
}

\begin{abstract}
In this study, an experimental investigation carried out to assess the performance of addition of an inert material on stabilization of an expansive soil in order to solve the lifting structures caused by this phenomenon. The results showed that an addition of glass is capable of reducing the actions of swelling. From the global analysis of the porosity using an ultrasonic device, we measure the propagation velocities of ultrasonic pulse through the tested clay samples. This reveals a change occurring in the porosity of the clay when glass is added to it. As a result some interesting conclusions concerning the expansive soil-material amendment are drawn.
\end{abstract}

Keywords: clay, swelling, glass, soil-additive

\section{Introduction}

En génie civil, le phénomène de retrait -gonflement de certains sols argileux et des formations géologiques argileuses induisent des tassements différentiels qui se manifestent par des désordres affectant principalement les structures construites en surfaces (bâtiments, fondations superficielles, ouvrages de soutènement, remblai...). Les exemples de désordres liés à la présence d'argile gonflante sont nombreux et variés.

Malgré ces inconvénients, les propriétés des argiles gonflantes; faible perméabilité, potentiel important de rétention, et bonne capacité d' auto cicatrisation en font des matériaux très intéressants dans de nombreuses applications, en particulier, en géotechnique de l'environnement pour la construction de barrières ouvragées destinées à confiner des colis de déchets nucléaires stockés en profondeur, pour l'élaboration de noyau de barrages en terre. Cependant ces matériaux peuvent être soumis à des variations de température et contrainte mécanique et hydraulique importantes qui peuvent mettre en cause leur bon fonctionnement.

Les argiles gonflantes sont donc présentes dans de nombreux contextes leurs propriétés pouvant être à la fois profitables et ou dommageables. Il est donc important d'appréhender le comportement hydromécanique de ces matériaux afin de mieux maîtriser leur utilisation.
En Algérie qui est un pays à climat contrasté, plusieurs cas de désordres liés au gonflement ont été recensés, la raffinerie de In-Amenas, l'hopital N'gaous (batna) [1,2], les lignes de chemin de fer (Jijel) [3], ... etc.

Plusieurs études ont été consacrées à ce phénomène afin de le maitriser. Néanmoins sa complexité n'a pas encore permis d'atteindre jusqu'à présent des solutions pouvant le résoudre.

De nombreuses relations empiriques ont été proposées pour caractériser l'expansibilité et la pression de gonflement lors de son hydratation. Ces relations expriment le gonflement sur la base de quelques caractéristiques du sol qui sont en principe simples à déterminer en laboratoire ([4]). Des études aussi nombreuses se sont intéressées aux facteurs intervenant dans le gonflement, $([4,5,6])$

Un bon nombre de solutions a été proposé afin de résoudre ce problème. Parmi ces solutions, il y a les techniques de stabilisation des sols. Parmi ces techniques, on a la stabilisation chimique, thermique, et la stabilisation des sols par additifs tels que l'apport de chaux et de ciment.

Plusieurs auteurs ont étudié l'influence de l'ajout de sable sur le gonflement $([7,8,9,10])$. Ces études confirment le rôle positif qu'a le sable sur la réduction du gonflement des sols expansifs et ce, par sa capacité d'amortir le gonflement à travers les vides importants qu'il contient. 
L'étude présentée ici a comme objectif d'analyser en laboratoire l'effet de la performance d'un ajout d'un matériau inerte similaire au sable -le verre-, sur le comportement d'un sol potentiellement gonflant utilisé dans des aspects différents.

\section{Caractéristiques géotechniques des matériaux utilisés}

Dans cette étude on a utilisé de la bentonite et des billes de verre.

\subsection{La bentonite}

La bentonite est un matériau argileux composé de plusieurs minéraux, principalement de la montmorillonite et d'un faible pourcentage de Kaolinite et d'Illite avec en général quelques traces de quartz.

La bentonite utilisée dans cette investigation provient de M'zila. C'est un matériau non traité, de couleur grise et se présente sous forme de poudre finement broyée. Le gisement de la bentonite de M'zila est situé au Nord-est de la ville de Mostaganem et à environ $10 \mathrm{Km}$ à l'est du village Achastas.

\subsubsection{Quelques propriétés de la bentonite}

L'analyse granulométrique a nécessité un tamisage à sec pour la distribution dimensionnelle en poids des particules supérieures ou égales à $80 \mu \mathrm{m}$, et une sédimentométrie pour la distribution dimensionnelle en poids des particules inférieures à $80 \mu \mathrm{m}$. Les résultats montrent que cette bentonite a été broyée très finement, le pourcentage des éléments de diamètre inférieur à $80 \mu \mathrm{m}$ est égale à $92 \%$ et les éléments de diamètre inférieur à $2 \mu \mathrm{m}$ sont évalués à $42 \%$.

Ses limites d'Atterberg qui sont la limite de liquidité, la limite de plasticité sont: $\mathrm{W}_{1}=187 \%, \mathrm{~W}_{\mathrm{p}}=$ $47 \%$. Son activité de skempton est de 3.33. On remarque la valeur très élevée de la limite de liquidité.

Cette bentonite a une grande affinité à l'eau. Elle est composée essentiellement de la silice (58\%) et d'alumine (19\%) qui sont des minéraux argileux favorisant le gonflement.

\subsection{Le verre}

Les billes utilisées dans cette étude sont en verre de forme sphérique de $1 \mathrm{~mm}$ de diamètre.

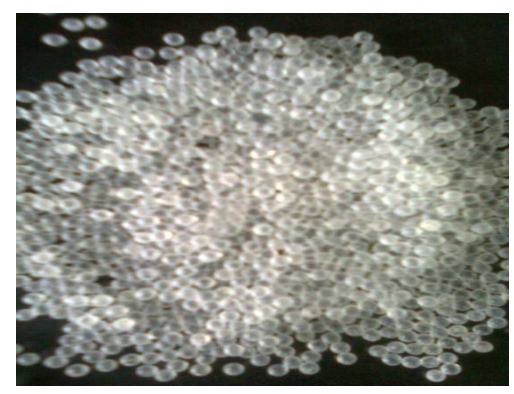

Fig. 1. Billes de verre utilisé
Des essais de compression à l'œdomètre effectués sur des billes de $5 \mathrm{~mm}$ de diamètre, ont montré que les billes ne tassent pas jusqu'à ce que la charge appliquée dépasse la limite de résistance à la compression des billes provoquant leur cassure et leur dégradation.

\section{Procédure expérimentale et tests réalisés}

\subsection{La consistance}

La consistance est un paramètre très important dans la reconnaissance d'un matériau, il nous informe sur son état et son comportement en fonction de son humidité.

Dans cette partie, on étudie l'influence de l'ajout de la poudre de verre sur la plasticité de la bentonite.

On a à déterminer les limites d'Atterberg (la limite de liquidité $\mathrm{W}_{\mathrm{L}}$, la limite de plasticité $\mathrm{W}_{\mathrm{P}}$ et l'indice de plasticité $I_{P}$.) de plusieurs échantillons correspondant à des dosages différents de poudre de verre.

La détermination de ces paramètres s'est faite suivant la NF P 94-051.

\subsection{Gonflement}

Une série d'essais a été menée afin d'étudier la performance d'un ajout de billes de verre $(\mathrm{d}=1 \mathrm{~mm})$ sur les caractéristiques de gonflement de la bentonite. Les échantillons ont été préparés à la température ambiante à une même teneur en eau de $26.5 \%$ et une même densité sèche 1.3 par malaxage et compactés statiquement et directement dans la trousse oedométrique à l'aide d'une presse manuelle.

\subsection{Porosité}

Le but de cette investigation est de tester la porosité des mélanges argile-poudre de verre à travers la vitesse de propagation des pulsations ultrasonores. On essaiera d'aboutir à quelques caractéristiques des pulsations liant le changement du milieu aux variations de la vitesse des pulsations ultrasonores.

Pour dérouler cette analyse, on a confectionné des échantillons cylindrique de bentonite seule et de bentonite + billes de verre de hauteur $\mathrm{h}=140 \mathrm{~mm}$ et de diamètre $\mathrm{d}=70 \mathrm{~mm}$ à une teneur en eau optimale $\left(\mathrm{W}_{\mathrm{opt}}=\right.$ $26,5 \%$ ) et une densité égale à 1.5 . Les échantillons ont été préparés par malaxage à l'aide d'un pétrin.

Les tests réalisés commencent par l'étalonnage de l'analyseur,

barreau de calibrage dont on connait les caractéristiques au préalable. Ensuite on mesure la vélocité d'un train d'onde ultrasonique, qui traverse les éprouvettes de sol testées

Pour garantir une bonne transmission des ondes dans le corps de l'éprouvette et avant d'ajuster le système de mesure, on applique des minces couches de graisses de contact sur les deux faces des transducteurs (émetteur et récepteur). Sur l'écran de l'analyseur sont représentés le temps ou la vitesse de transit de l'onde, cela selon la configuration de l'analyseur. 


\section{Présentation et discussion des résultats}

\subsection{Variation de la limite de liquidité}

Les résultats de la variation de la limite de liquidité de la bentonite avec la teneur en poudre de verre sont représentés dans la courbe suivante.

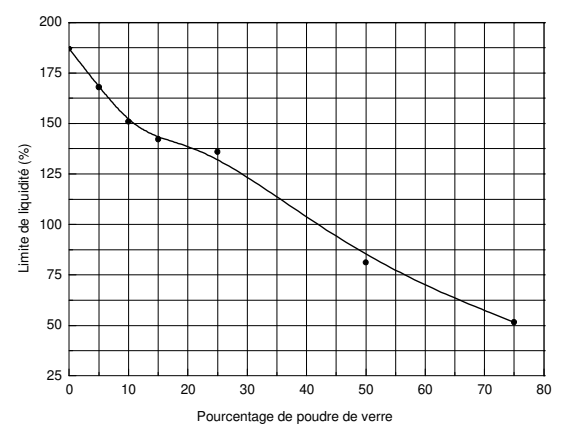

Fig.2. Variation de la limite de liquidité en fonction de la teneur en poudre de verre

En analysant ces résultats, on constate une diminution de la limite de la liquidité avec l'augmentation de la teneur en poudre de verre. Cette chute est d'abord très rapide pour de faibles teneurs en poudre, puis elle est régulière pour des teneurs moyennes mais moins importantes que la précédente et devient enfin très faible pour de très fortes teneurs en poudrette de verre des mélanges. Ces phases de variation peuvent être le résultat du changement du comportement des mélanges par ajout de matériaux inertes d'un sol argileux très plastique à un sol faiblement plastique pour de très fortes quantités de poudrette de verre ajoutée. Cette réduction est due à la diminution de la teneur en éléments fins actifs qui contribuent à la plasticité.

En outre, ces résultats montrent qu'il n'y a pas une liaison claire entre le dosage en poudre de verre et la limite de liquidité, ce qui dicte qu'il existe d'autres facteurs autres que celui de la diminution de la fraction argileuse, il s'agit aussi de la diminution de la surface spécifique d'adsorption d'eau et la réduction du nombre de liaisons entre les particules argileuses qui contribuent à la réduction de cette limite de liquidité.

\subsection{Variation de la limite de plasticité}

Les résultats de la variation de la limite de plasticité de la bentonite avec la teneur en poudre de verre sont représentés dans la courbe de la figure 3 suivante.

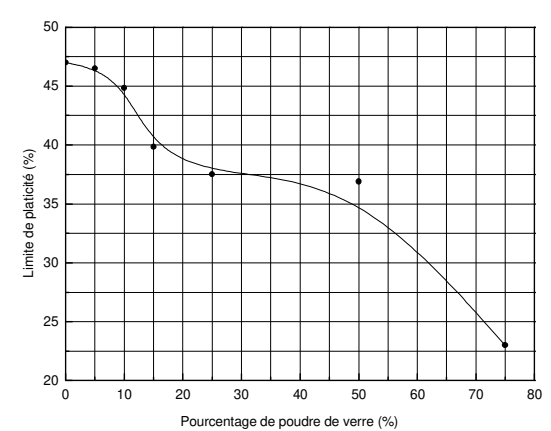

Fig.3. Variation de la limite de plasticité en fonction de la teneur en poudre de verre

On constate de cette courbe une diminution de la limite de la plasticité avec la teneur en poudre de verre. Cette réduction est serrée comparée à la limite de liquidité, mais elle est aussi due à la diminution de la teneur en éléments fins actifs et l'augmentation de la quantité du matériau inerte. Elle confirme aussi le changement de comportement du matériau à très fortes teneurs en poudrette de verre.

\subsection{Variation de l'indice de plasticité}

Les résultats de la variation de l'indice de plasticité de la bentonite avec la teneur en poudre de verre sont représentés dans la courbe suivante.

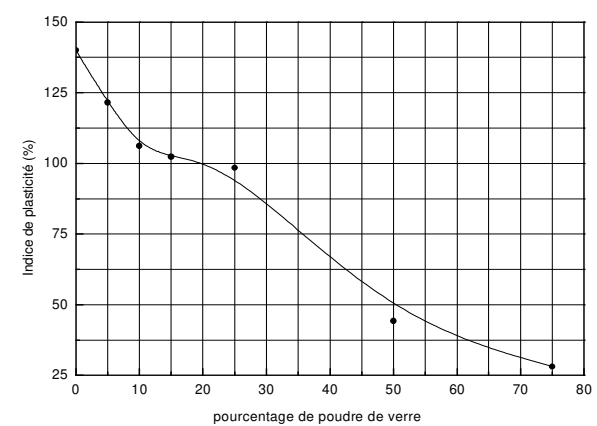

Fig.4. Variation de l'indice de plasticité en fonction du pourcentage en poudre de verre

La courbe de la figure 4 montre une diminution de l'indice de plasticité avec la teneur en poudre de verre. Cette réduction est due à la diminution des limites de liquidité et de plasticité. Son taux de réduction a été important du fait que la diminution de la limite de liquidité est plus importante que celle de la limite de plasticité, et puisque l'indice de plasticité n'est rien d'autre que la différence entre ces deux limites, alors sa décroissance a été importante.

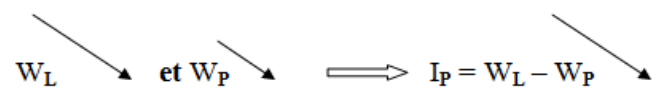

Cette diminution de l'indice de plasticité engendre la réduction de l'étendue du domaine plastique, ce qui confirme le passage d'un matériau très plastique vers un matériau moins plastique et moins gonflants par conséquent. 


\subsection{Gonflement}

Les résultats des tests effectués dans cette investigation, donnée dans les figures $5,6,7$ et 8 appellent les observations suivantes : On constate que les deux paramètres de gonflement diminuent avec le pourcentage de billes additionné

Le taux de réduction de la pression de gonflement est de $24 \%, 66 \%$ et $94 \%$ pour dosages de $25 \%, 50 \%$ et $75 \%$ respectivement., On constate aussi que la variation du gonflement avec la teneur en verre n'est pas linéaire, montrant que la diminution du gonflement n'est pas que le résultat de la diminution de la fraction argileuse, d'autres facteurs contribuent à cette réduction qui est la réduction des liaisons bentonite-bentonite engendrée par le surplus de billes et la dispersion des particules de bentonite dans le mélange.

En revanche, la figure 8 montre l'évolution de la teneur en eau finale avec la teneur en billes de verre qui est due à la réduction de la surface spécifique globale d'adsorption d'eau. On remarque que le taux de réduction n'est pas aussi important comparée à celui de la pression et le potentiel de gonflement, cela est dû à la participation des billes de verre dans la préservation de l'eau par adsorption.

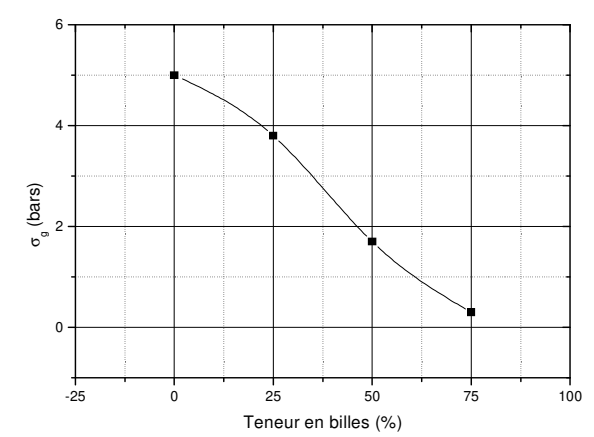

Fig.5. Variation de la pression avec la teneur en billes de verre

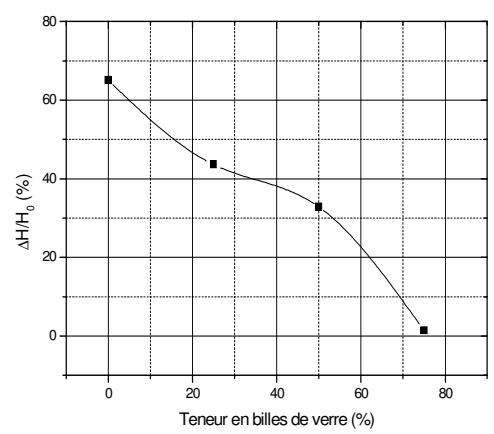

Fig.6. Variation du taux de gonflement en fonction de la teneur en billes de verre

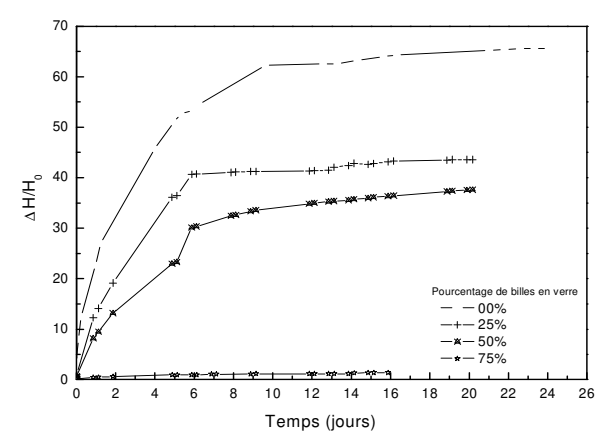

Fig.7.Evolution de la cinétique de gonflement de la bentonite seule et des mélanges bentonite - billes en verre

Les courbes de gonflement en fonction du temps de la bentonite mélangée aux billes de verre sont représentées par la figure 7. On remarque que la tendance de la variation du gonflement dans le temps est la même pour les différentes concentrations. Ces courbes montrent un potentiel de gonflement plus élevé pour la bentonite seule et qui diminue avec l'augmentation du dosage en billes de verre des mélanges.

Sur chaque courbe, on distingue que l'évolution du potentiel de gonflement se produit en deux temps. Dans un premier temps le gonflement est très rapide, il démarre dès que l'échantillon est inondé. Cette première étape de gonflement correspond à un gonflement macroscopique régi principalement par la perméabilité du milieu et le gradient de succion. Dans un deuxième temps le gonflement devient lent et progressif (gonflement microscopique) jusqu'à atteindre la durée nécessaire à la stabilisation. Cette dernière se raccourcit avec l'augmentation de la teneur en billes de verre des mélanges. La deuxième phase de gonflement constituerait le processus d'hydratation progressive des minéraux argileux et des cations adsorbés, cette hydratation s'effectuant à partir de l'eau qui est disponible dans les pores. On remarque aussi que l'ajout des billes de verre réduit aussi le temps de stabilisation du gonflement des mélanges. Plus exactement la première phase qui correspond au gonflement primaire a tendance à disparaitre à $75 \%$. Ce changement dans la cinétique du gonflement peut être dû en grande partie, au changement dans la perméabilité des échantillons. Celle-ci augmente suite au traitement par ajout de billes. Cette nouvelle structure du sol aide l'eau à s'infiltrer rapidement et remplir les gros pores.

En revanche, la figure 8 montre l'évolution de la teneur en eau finale avec la teneur en billes de verre qui est due à la réduction de la surface spécifique globale d'adsorption d'eau. On remarque que le taux de réduction n'est pas aussi important comparée à celui de la pression et le potentiel de gonflement, cela est dû à la participation des billes de verre dans la préservation de l'eau par adsorption. 


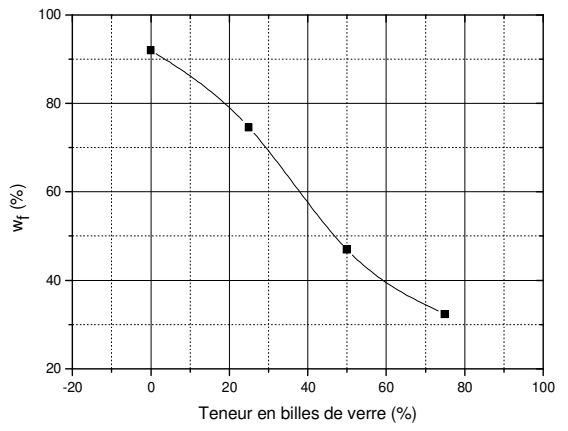

Fig.8. Variation de la teneur en eau finale en fonction de la teneur en billes de verre

\subsection{Evolution de la vitesse de propagation des ondes ultrasonores}

La figure 9 montre la diminution de la vitesse de propagation des ondes ultrasonores par ajout de billes de verre, pour devenir très faible et incontrôlable à des teneurs élevées. On signale qu'à 50\% d'ajout, le sol étudié devient très poreux et la vitesse devient très variable, l'appareil affiche en effet plusieurs valeurs de vitesse. Ces dernières sont tous faibles tendant vers zéro.

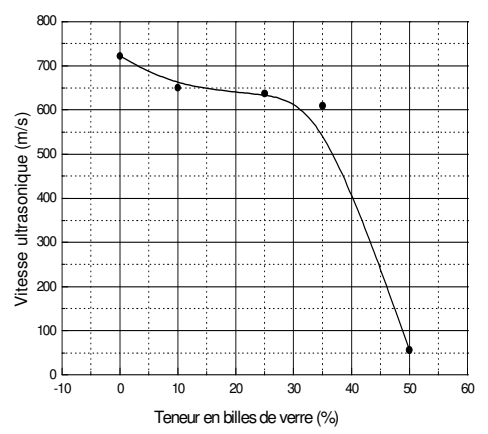

Fig. 9. Vitesse et teneur en billes de verre

Cette variation dans le sens décroissant exprime l'augmentation de la porosité des mélanges par ajout de billes en verre. Ce résultat permet de mettre en évidence la variation dans la structure du sol traité et de répondre au pourquoi de la diminution du gonflement par ajout de ce matériau.

\section{Conclusion}

La présente étude comporte des essais géotechniques standards, a étudié comment agit l'additif (le verre) pour réduire le gonflement d'une bentonite. Elle a permis d'asseoir des conclusions intéressantes qu'on résume dans ce qui suit.

1. La poudre de verre a un effet réducteur sur tous les paramètres de consistance. Cette réduction est élevée pour de faibles teneurs en poudre et diminue pour des teneurs élevées.

2. Le potentiel et la pression de gonflement de la bentonite traitée avec billes de verre diminuent avec le dosage en billes. Cette réduction semble être plus importante que le dosage ajouté. Le non linéarité de la diminution est dû à la diminution au départ la fraction argileuse par ajout de la poudre du verre et à la diminution de la densité de l'argile.

3. Enfin l'analyse globale de la porosité par propagation de pulsations ultrasoniques, a montré une diminution de la vitesse. Cette variation dans le sens décroissant exprime l'augmentation de la porosité des mélanges par ajout des billes de verre. Ce résultat met en évidence la diminution de la densité de l'argile par ajout de billes de verre.

4. Ces constatations confirment les relations déjà établies entre la consistance, la porosité et le comportement des sols vis-à-vis du gonflement. Il en ressort que la réduction du gonflement d'un sol est engendrée par la rupture des liaisons entre les particules argileuses et la création des pores suite à l'incorporation du matériau d'amendement qui est le verre.

5. Les résultats obtenus encouragent à approfondir l'étude et à réfléchir peut-être à d'autres techniques pour lutter contre le phénomène de gonflement des sols, par exemple, partant d'un esprit écologique, l'exploitation des déchets industriels dans la stabilisation du gonflement des sols.

\section{References}

[1] Z. Derriche et M. Kebaili, "Prévision du gonflement des argiles d'In Amenas," Bulletin des Laboratoires des ponts et Chaussées, pp. 15-23, (1998)

[2] A. Hachichi and J. M. Fleureau, "Caratérisation de Quelques Sols Gonflants d'Algérie," Revue Française de Géotechnique, No. 86, pp. 37-51, (1999).

[3] M. Lamara and A. Merah, "Analyse d'un Cas Pat logique de Gonflement de Sol (Université de Laghouat)," Journée d'Études sur les Sols Gonflants, Tlemcen, 27-28 Octobre 2002, pp. 101-110.

[4] M.A. El-Sobhy \& A. El-Sayed ''Some factors affecting swelling of clayey soils'. Faculty of Engineering Department, University of Al-Azhar, Cairo, Egypt (1981).

[5] F.H. Chen, Foundation on expansive soil. Elsevier edition. (1988)

[6] A. Djedid, and N. Ouadah,, Paramètres d'état et gonflement des sols argileux, exemple de la teneur en eau et de la densité sèche initiale. Colloque international sols non saturés et environnement. pp.436-444 (2009)..

[7] B. Louafi,, Analyse de Quelques Mécanismes physiques de la stabilisaton des sols expensifs par additifs à aspect inerte. Thèse de doctorat , Université des Sciences et la Technologie Houari Boumediènne, Alger, Algerie (2013).

[8] H.A. Alawadiji,. Swell and compressibility characteristics of sand-bentonite mixtures inundated with liquids. Applied Clay Science, 15(3-4), 411-430 (1999).

[9] L. Bengraa , A. Hachichi A., S.A Bourokba., \& A. Benaissa, Etude de la stabilisation des argiles gonflantes par ajout de sable de carrière. 2éme Journée d'études sur les sols gonflants. Université de Tlemcen (Algérie), pp. 101- 112 (2005).

[10] B. Louafi \& R. Bahar, SAND: An Additive for Stabilzation of Swelling Clay Soils, International Journal of Geosciences, 2012, Vol.3.pp.719-725 (2012). 